MSC 97M50, 74J15, 74J30, 74A99

DOI: $10.14529 / \mathrm{mmp} 150403$

\title{
MATHEMATICAL MODELLING OF WAVY SURFACE OF LIQUID FILM FALLING DOWN A VERTICAL PLANE AT MODERATE REYNOLDS' NUMBERS
}

\author{
L.A. Prokudina, South Ural State University, Chelyabinsk, Russian Federation, \\ prokudina-la@mail.ru, \\ Ye.A. Salamatov, Emerson Process Management, Metran, Chelyabinsk, Russian \\ Federation, salamatov@gmx.com, yevgeny.salamatov@emerson.com
}

\begin{abstract}
Development of periodic disturbances on free surface of water film falling down vertical plane for Reynolds' number $R e \in[5 ; 10]$ is investigated. The investigation is implemented in a scope of the nonlinear differential equation for evolution of free surface of falling down liquid film. The equation is solved by a finite differencies method at rectangular uniformly spaced grid. By researching the growth of unit inaccuracy, the conditions on parameters of computation grid for inaccuracies to be not increasing are obtained. As a result, waveforms of water film, time spent to form the regular wave mode and amplitudes of periodic disturbances are calculated. Calculated amplitudes and experimental ones are compared.
\end{abstract}

Keywords: liquid film; amplitude; waveform; nonlinear evolution of disturbances.

\section{Introduction}

Liquid film flows (thin layers of viscous fluids) are implemented in many industries such as chemical, oil, energy and others. Advantages of liquid film flows are energy efficiency, greater product purity etc. These advantages are determined by highly extended contact line between streams moving and interacting each other. The contact line is formed by waves on free surface of liquid film.

Wavy flows of liquid films have been investigated since the middle of the $20^{\text {th }}$ century $[1$, 2]. Nevertheless they are still interesting [3-10]. Their study is requiered by both practical (due to variety of environments where liquid film flows are realised) and theoretical reasons (since film flows are described by nonlinear partial differential equations).

\section{The Model}

Consider a thin layer of wavy liquid film falling down a vertical smooth plane under the action of gravity. The liquid is supposed to be viscous, uniform and incompressible. It is interacting with a gas streaming in parallel to the plane. The gas creates constant tangential stress $\bar{\tau}$ on the free surface of the liquid film (Fig. 1). If the liquid and the gas are moving in one direction, cocurrent mode is implemented, otherwise countercurrent mode is realised.

The equation

$$
\begin{aligned}
\frac{\partial \psi}{\partial t} & =b_{1} \frac{\partial^{4} \psi}{\partial x^{4}}+b_{2} \frac{\partial^{2} \psi}{\partial x^{2}}+b_{3} \frac{\partial \psi}{\partial x}+b_{4} \frac{\partial^{2} \psi}{\partial x \partial t}+b_{5} \psi \frac{\partial \psi}{\partial x}+b_{6} \psi \frac{\partial^{2} \psi}{\partial x^{2}}+b_{7} \psi \frac{\partial^{4} \psi}{\partial x^{4}}+b_{8} \psi \frac{\partial^{2} \psi}{\partial x \partial t}+ \\
& +b_{9} \frac{\partial \psi}{\partial x} \frac{\partial \psi}{\partial t}+b_{10}\left(\frac{\partial \psi}{\partial x}\right)^{2}+b_{11} \frac{\partial \psi}{\partial x} \frac{\partial^{3} \psi}{\partial x^{3}}+b_{12} \psi^{2} \frac{\partial \psi}{\partial x}+b_{13} \psi^{2} \frac{\partial^{2} \psi}{\partial x^{2}}+b_{14} \psi \frac{\partial \psi}{\partial x} \frac{\partial^{3} \psi}{\partial x^{3}}
\end{aligned}
$$




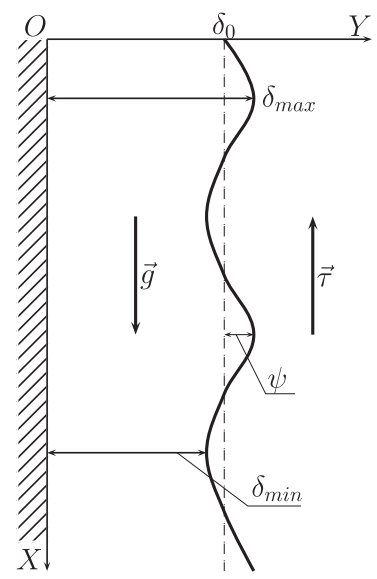

Fig. 1. Definition sketch

describes evolution of liquid film free surface [10-12]. Here $b_{1}=-\frac{\sigma R e}{3}, b_{2}=\frac{3}{40} R e^{3} F(F-$ $\tau), b_{3}=-\operatorname{Re}(F-\tau), b_{4}=\frac{5}{24} R e^{2} F, b_{5}=-2 R e F+R e \tau, b_{6}=-\frac{3}{8} R e^{3} F \tau+\frac{9}{20} R e^{3} F^{2}$, $b_{7}=3 b_{1}, b_{8}=4 b_{4}, b_{9}=b_{8}, b_{10}=b_{6}, b_{11}=3 b_{1}, b_{12}=-R e F, b_{13}=-\frac{3}{4} R e^{3} F \tau+$ $\frac{9}{8} R e^{3} F^{2}, b_{14}=6 b_{1}$, Re is the Reynolds number, $F$ is the Froude number, $\sigma$ is the surface tension (unitless), $\tau$ is the shear stress (unitless). The required function $\psi(x, t)$ denotes the displacement of free surface (Fig. 1) from its undisturbed position $\delta_{0}\left(\psi \ll \delta_{0}\right)$. We assume $\tau>0$ for countercurrent mode and $\tau<0$ for cocurrent one. If $\tau=0$, free mode is implemented.

\section{Searching for Periodical Solution to the Governing Equation}

A periodical solution to $(1)$ is searched numerically by finite defferencies method. We consider a rectangular domain $\Omega=\left\{(x, t): x_{\text {begin }} \leqslant x \leqslant x_{\text {end }}, 0 \leqslant t<t_{\text {end }}\right\}$. Let us cover $\Omega$ by a uniform grid $\left(x_{i}, t_{j}\right)$. The grid has a step $\Delta x=\frac{x_{\text {end }}-x_{\text {begin }}}{N_{x}}$ along the spacial variable $x$ and has a step $\Delta t$ along the time variable $t$. In this case $x_{i}=x_{\text {begin }}+i \Delta x$, $t_{j}=j \Delta t\left(i=0,1, \ldots N_{x} ; j=0,1,2, \ldots\right)$. By $\psi_{i}^{j}$ denote $\psi\left(x_{i}, t_{j}\right)$. The derivatives in $(1)$ are approximated as follows

$$
\begin{aligned}
& \left.\frac{\partial^{4} \psi}{\partial x^{4}}\right|_{i} ^{j} \approx \frac{\psi_{i+2}^{j}-4 \psi_{i+1}^{j}+6 \psi_{i}^{j}-4 \psi_{i-1}^{j}+\psi_{i-2}^{j}}{\Delta x^{4}}=\frac{d_{4, i}^{j}}{\Delta x^{4}}, \\
& \left.\frac{\partial^{3} \psi}{\partial x^{3}}\right|_{i} ^{j} \approx \frac{\psi_{i+2}^{j}-3 \psi_{i+1}^{j}+3 \psi_{i-1}^{j}-\psi_{i-2}^{j}}{2 \Delta x^{3}}=\frac{d_{3, i}^{j}}{\Delta x^{3}}, \\
& \left.\frac{\partial^{2} \psi}{\partial x^{2}}\right|_{i} ^{j} \approx \frac{\psi_{i+1}^{j}-2 \psi_{i}^{j}+\psi_{i-1}^{j}}{\Delta x^{2}}=\frac{d_{2, i}^{j}}{\Delta x^{2}} \\
& \left.\frac{\partial \psi}{\partial x}\right|_{i} ^{j} \approx \frac{\psi_{i+1}^{j}-\psi_{i-1}^{j}}{2 \Delta x}=\frac{d_{1, i}^{j}}{\Delta x}
\end{aligned}
$$




$$
\begin{aligned}
\left.\frac{\partial \psi}{\partial t}\right|_{i} ^{j} & \approx \frac{\psi_{i}^{j+1}-\psi_{i}^{j}}{\Delta t}, \\
\left.\frac{\partial^{2} \psi}{\partial x \partial t}\right|_{i} ^{j} & \approx \frac{1}{\Delta t}\left(\frac{\psi_{i+1}^{j+1}-\psi_{i}^{j+1}}{\Delta x}-\frac{\psi_{i+1}^{j}-\psi_{i}^{j}}{\Delta x}\right) .
\end{aligned}
$$

Substituting (2) - (7) in to (1) and modifying it, we obtain

$$
\begin{aligned}
& \psi_{i}^{j+1}(\left.\frac{1}{\Delta t}+\frac{b_{4}}{\Delta t \Delta x}+\frac{b_{8} \psi_{i}^{j}}{\Delta t \Delta x}-\frac{b_{9} d_{1, i}^{j}}{\Delta t \Delta x}\right)+\psi_{i+1}^{j+1}\left(-\frac{b_{4}}{\Delta t \Delta x}-\frac{b_{8} \psi_{i}^{j}}{\Delta t \Delta x}\right)= \\
&=b_{1} \frac{d_{4, i}^{j}}{\Delta x^{4}}+b_{2} \frac{d_{2, i}^{j}}{\Delta x^{2}}+b_{3} \frac{d_{1, i}^{j}}{\Delta x}+b_{5} \psi_{i}^{j} \frac{d_{1, i}^{j}}{\Delta x}+b_{6} \psi_{i}^{j} \frac{d_{2, i}^{j}}{\Delta x^{2}}+b_{7} \psi_{i}^{j} \frac{d_{4, i}^{j}}{\Delta x^{4}}+b_{10} \frac{\left(d_{2, i}^{j}\right)^{2}}{\Delta x^{2}}+ \\
&+b_{11} \frac{d_{1, i}^{j}}{\Delta x} \frac{d_{3, i}^{j}}{\Delta x^{3}}+b_{12}\left(\psi_{i}^{j}\right)^{2} \frac{d_{1, i}^{j}}{\Delta x}+b_{13}\left(\psi_{i}^{j}\right)^{2} \frac{d_{2, i}^{j}}{\Delta x^{2}}+b_{14} \psi_{i}^{j} \frac{d_{1, i}^{j}}{\Delta x} \frac{d_{3, i}^{j}}{\Delta x^{3}}+\frac{\psi_{i}^{j}}{\Delta t}- \\
& \quad-\frac{b_{4}}{\Delta t} \frac{\psi_{i+1}^{j}-\psi_{i}^{j}}{\Delta x}-\frac{b_{8} \psi_{i}^{j}}{\Delta t} \frac{\psi_{i+1}^{j}-\psi_{i}^{j}}{\Delta x}-\frac{b_{9} d_{1, i}^{j} \psi_{i}^{j}}{\Delta t \Delta x}, \quad 0 \leqslant i \leqslant N_{x} .
\end{aligned}
$$

Assume

$$
\Delta t=\eta \Delta x^{3}, \quad \eta>0,
$$

and, multiplying both sides of (8) by $\eta \Delta x^{4}$, we get

$$
\left(\alpha_{i}^{j}-\beta_{i}^{j}\right) \psi_{i}^{j+1}+\beta_{i}^{j} \psi_{i+1}^{j+1}=\eta \gamma_{i}^{j}+\left(\alpha_{i}^{j}-\beta_{i}^{j}\right) \psi_{i}^{j}+\beta_{i}^{j} \psi_{i+1}^{j}, \quad 0 \leqslant i \leqslant N_{x},
$$

where $\alpha_{i}^{j}=\Delta x-b_{9} d_{1, i}^{j}, \beta_{i}^{j}=-b_{4}-b_{8} \psi_{i}^{j}, \gamma_{i}^{j}=b_{1} d_{4, i}^{j}+b_{2} d_{2, i}^{j} \Delta x^{2}+b_{3} d_{1, i}^{j} \Delta x^{3}+b_{5} \psi_{i}^{j} d_{1, i}^{j} \Delta x^{3}+$ $b_{6} \psi_{i}^{j} d_{2, i}^{j} \Delta x^{2}+b_{7} \psi_{i}^{j} d_{4, i}^{j}+b_{10}\left(d_{1, i}^{j}\right)^{2} \Delta x^{2}+b_{11} d_{1, i}^{j} d_{3, i}^{j}+b_{12}\left(\psi_{i}^{j}\right)^{2} d_{1, i}^{j} \Delta x^{3}+b_{13}\left(\psi_{i}^{j}\right)^{2} d_{2, i}^{j} \Delta x^{2}+$ $b_{14} \psi_{i}^{j} d_{1, i}^{j} d_{3, i}^{j}$.

Thus, in order to search a solution to (1) it is necessary to solve the system of equations (10).

Further, consider how computational inaccuracies behave while transferring from the $j^{\text {th }}$ 'time layer' to the $(j+1)^{\text {th }}$ one. We will do that using a method of a unit inaccuracy growth as described in [13].

So, we assume that in any node $(i, j)$ the function $\psi_{i}^{j}$ contains inaccuracy $\varepsilon_{i}^{j}$ (we suppose $\left.\varepsilon_{i}^{j} \ll \psi_{i}^{j}\right)$. But for the other nodes on the $j^{\text {th }}$ 'time layer', we assume that the function $\psi_{i}^{j}$ does not contain any inaccuracies (viz it is calculated exactly). For the node $(i, j)$, it can be written that $\psi_{i}^{j}=\bar{\psi}_{i}^{j}+\varepsilon_{i}^{j}$, here $\bar{\psi}_{i}^{j}$ marks exactly calculated part of the function $\psi$ (further, we denote by 'bar' any variable calculated exactly).

The inaccuracy $\varepsilon_{i}^{j}$ leads to appearing of inaccuracies in the finite differencies $(2)-(7)$ for the nodes $(i-2, j),(i-1, j),(i, j),(i+1, j),(i+2, j)$ as follows

\begin{tabular}{c|c|c|c|c|c} 
& $(i-2, j)$ & $(i-1, j)$ & $(i, j)$ & $(i+1, j)$ & $(i+2, j)$ \\
\hline$d_{1}$ & $\bar{d}_{1, i-2}^{j}$ & $\bar{d}_{1, i-1}^{j}+\frac{\varepsilon_{i}^{j}}{2}$ & $\bar{d}_{1, i}^{j}$ & $\bar{d}_{1, i+1}^{j}-\frac{\varepsilon_{i}^{j}}{2}$ & $\bar{d}_{1, i+2}^{j}$ \\
\hline$d_{2}$ & $\bar{d}_{2, i-2}^{j}$ & $\bar{d}_{2, i-1}^{j}+\varepsilon_{i}^{j}$ & $\bar{d}_{2, i}^{j}-2 \varepsilon_{i}^{j}$ & $\bar{d}_{2, i+1}^{j}+\varepsilon_{i}^{j}$ & $\bar{d}_{2, i+2}^{j}$ \\
\hline$d_{3}$ & $\bar{d}_{3, i-2}^{j}+\frac{\varepsilon_{i}^{j}}{2}$ & $\bar{d}_{3, i-1}^{j}-\frac{3 \varepsilon_{i}^{j}}{2}$ & $\bar{d}_{3, i}^{j}$ & $\bar{d}_{3, i+1}^{j}+\frac{3 \varepsilon_{i}^{j}}{2}$ & $\bar{d}_{3, i+2}^{j}-\frac{\varepsilon_{i}^{j}}{2}$ \\
\hline$d_{4}$ & $\bar{d}_{4, i-2}^{j}+\varepsilon_{i}^{j}$ & $\bar{d}_{4, i-1}^{j}-4 \varepsilon_{i}^{j}$ & $\bar{d}_{4, i}^{j}+6 \varepsilon_{i}^{j}$ & $\bar{d}_{4, i+1}^{j}-4 \varepsilon_{i}^{j}$ & $\bar{d}_{4, i+2}^{j}+\varepsilon_{i}^{j}$
\end{tabular}




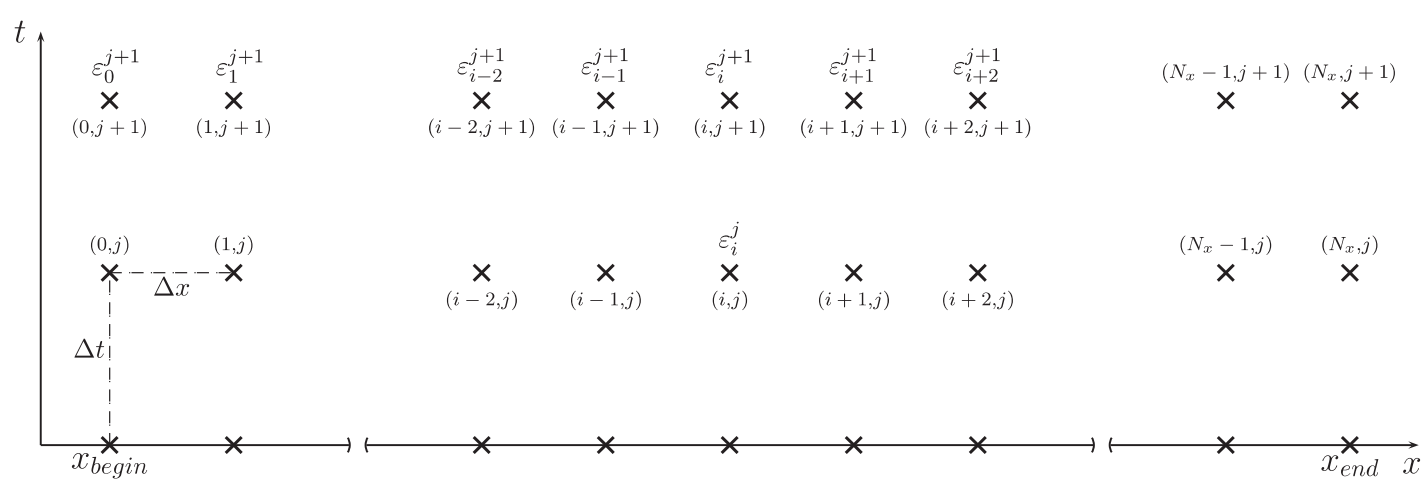

Fig. 2. The sketch of $\Omega$

And then, inaccuracies (11) being on the $j^{\text {th }}$ 'time layer' lead to inaccuracies on the $(j+1)^{\text {th }}$ 'time layer' as depicted on Fig. 2. For example, Equation (10) written for the node $(i+2, j+1)$ has the form

$$
\begin{aligned}
&\left(\bar{\alpha}_{i+2}^{j}-\bar{\beta}_{i+2}^{j}\right)\left(\bar{\psi}_{i+2}^{j+1}+\varepsilon_{i+2}^{j+1}\right)+\bar{\beta}_{i+2}^{j} \bar{\psi}_{i+3}^{j+1}= \\
&=\eta\left(\bar{\gamma}_{i+2}^{j}+b_{1} \varepsilon_{i}^{j}+b_{7} \bar{\psi}_{i+2}^{j} \varepsilon_{i}^{j}-b_{11} \bar{d}_{1, i+2}^{j} \frac{\varepsilon_{i}^{j}}{2}+b_{14} \bar{\psi}_{i+2}^{j} \bar{d}_{1, i+2}^{j} \frac{\varepsilon_{i}^{j}}{2}\right)+ \\
&+\left(\bar{\alpha}_{i+2}^{j}-\bar{\beta}_{i+2}^{j}\right) \bar{\psi}_{i+2}^{j}+\bar{\beta}_{i+2}^{j} \bar{\psi}_{i+3}^{j} .
\end{aligned}
$$

By $\varepsilon_{i+2}^{j+1}$ we denote the inaccuracy in the node $(i+2, j+1)$.

Taking into account $\psi_{i}^{j} \ll \delta_{0}$ and $\varepsilon_{i}^{j} \ll \psi_{i}^{j}$, modify Equation (12) as follows

$$
\begin{gathered}
\left(\Delta x+b_{4}\right) \varepsilon_{i+2}^{j+1} \approx \eta b_{1} \varepsilon_{i}^{j}, \\
\varepsilon_{i+2}^{j+1} \approx \kappa_{i+2} \varepsilon_{i}^{j},
\end{gathered}
$$

and

$$
\kappa_{i+2}=\eta \frac{b_{1}}{\Delta x+b_{4}} .
$$

Similarly, missing manipulations due to their tedious, we find constants of proportionality for the nodes $(i+1, j+1),(i, j+1),(i-1, j+1),(i-2, j+1)$ :

$$
\begin{gathered}
\kappa_{i+1}=\eta\left(\frac{-4 b_{1}+b_{2} \Delta x^{2}-\frac{b_{3}}{2} \Delta x^{3}}{\Delta x+b_{4}}+\frac{b_{1} b_{4}}{\left(\Delta x+b_{4}\right)^{2}}\right), \\
\kappa_{i}=1+\frac{\eta\left(6 b_{1}-2 b_{2} \Delta x^{2}\right)}{\Delta x+b_{4}}+\frac{b_{4}}{\Delta x+b_{4}} \kappa_{i+1}, \\
\kappa_{i-1}=\frac{\eta\left(-4 b_{1}+b_{2} \Delta x^{2}+\frac{b_{3}}{2} \Delta x^{3}\right)-b_{4}+b_{4} \kappa_{i}}{\Delta x+b_{4}}, \\
\kappa_{i-2}=\frac{\eta\left(6 b_{1}-2 b_{2} \Delta x^{2}\right)+b_{4} \kappa_{i-1}}{\Delta x+b_{4}} .
\end{gathered}
$$

Equations in $(10)$ for the nodes $(l, j+1), 0 \leqslant l \leqslant i-3$, have the form $\left(\bar{\alpha}_{l}^{j}-\bar{\beta}_{l}^{j}\right)\left(\bar{\psi}_{l}^{j+1}+\varepsilon_{l}^{j+1}\right)+\bar{\beta}_{l}^{j}\left(\bar{\psi}_{l+1}^{j+1}+\varepsilon_{l+1}^{j+1}\right)=\eta \bar{\gamma}_{l}^{j}+\left(\bar{\alpha}_{l}^{j}-\bar{\beta}_{l}^{j}\right) \bar{\psi}_{l}^{j}+\bar{\beta}_{l}^{j} \bar{\psi}_{l+1}^{j}, \quad 0 \leqslant l \leqslant i-3$. 
Thus,

$$
\begin{gathered}
\varepsilon_{l}^{j+1} \approx \frac{b_{4}}{\Delta x+b_{4}} \varepsilon_{l+1}^{j+1}, \quad 0 \leqslant l \leqslant i-3, \\
\varepsilon_{l}^{j+1} \approx \frac{b_{4}}{\Delta x+b_{4}} \kappa_{l+1} \varepsilon_{i}^{j}, \quad 0 \leqslant l \leqslant i-3, \\
\kappa_{l}=\frac{b_{4}}{\Delta x+b_{4}} \kappa_{l+1}, \quad 0 \leqslant l \leqslant i-3 .
\end{gathered}
$$

The formulae (13) - (18) connect the unit inaccuracy $\varepsilon_{i}^{j}$ with ones appearing on the $(j+1)^{\text {th }}$ "time layer". Obviously, the inaccuracies on the $(j+1)^{\text {th }}$ 'time layer' will not increase if parameters $\eta$ and $\Delta x$ are chosen so that $\left|\kappa_{q}\right|<1,0 \leqslant q \leqslant i+2$. According to the formula (18), we get $\left|\kappa_{0}\right|<\cdots<\left|\kappa_{l}\right|<\cdots<\left|\kappa_{i-3}\right|<\left|\kappa_{i-2}\right|$. Therefore we may require only

$$
\left|\kappa_{q}\right|<1, \quad i-2 \leqslant q \leqslant i+2 .
$$

Unequalities (19) can be written with respect to $\eta$ as follows

$$
\eta<\frac{\eta<\frac{\Delta x+b_{4}}{\left|b_{1}\right|}=\eta_{1},}{\eta<\frac{\left(\Delta x+b_{4}\right)^{2}}{\left|3 b_{1} b_{4}+4 b_{1} \Delta x-b_{2} b_{4} \Delta x^{2}+\left(4 b_{1}+\frac{b_{3} b_{4}}{2}\right) \Delta x^{3}+\frac{b_{3}}{2} \Delta x^{4}\right|}=\eta_{2}}=\frac{2\left(\Delta x+b_{4}\right)^{3}}{\left|3 b_{1} b_{4}^{2}+8 b_{1} b_{4} \Delta x+\left(6 b_{1}-b_{2} b_{4}^{2}\right) \Delta x^{2}-\left(3 b_{2} b_{4}+\frac{b_{3} b_{4}^{2}}{2}\right) \Delta x^{3}-\left(2 b_{2}+\frac{b_{3} b_{4}}{2}\right) \Delta x^{4}\right|}=\eta_{3},
$$

Finally, we obtain the next condition

$$
\eta<\min \left(\eta_{1}, \eta_{2}, \eta_{3}, \eta_{4}, \eta_{5}\right)
$$

\section{Results}

Considere an initial periodical disturbance

$$
\psi(x, 0)=a_{0} \cdot \cos (k x)
$$


Table

Grid parameters

\begin{tabular}{|l|l|l|l|}
\hline$R e$ & $k$ & $\Delta x$ & $\eta \times 10^{-3}$ \\
\hline 5 & 0,07246 & 0,270 & 1,11 \\
\hline 6 & 0,08311 & 0,273 & 0,94 \\
\hline 7 & 0,09332 & 0,276 & 0,78 \\
\hline 8 & 0,10211 & 0,273 & 0,62 \\
\hline 9 & 0,10976 & 0,282 & 0.49 \\
\hline 10 & 0,11629 & 0,274 & 0,37 \\
\hline
\end{tabular}

that is developed on free surface of water film falling down a vertical plane. Disturbance (21) has the amplitude $a_{0}=10^{-4} \ll 1$ and the wave number $k$ (see Table). We consider $R e \in[5 ; 10]$ and $\tau=0$.

Put $\eta=\frac{1}{3} \min \left(\eta_{1}, \eta_{2}, \eta_{3}, \eta_{4}, \eta_{5}\right)$. The grid parameters are placed in Table. The length of $\left[x_{\min } ; x_{\max }\right]$ is multiple of 30 wave lengths of $(21)$. Calculations were carried out untill $t_{\text {end }}=1000$.

Our calculations demonstrate, that the regular wavy flows are formed on free surface of vertical water film. The waveforms are shown on Fig. 3 (we illustrated only one period and the $x$ axes was scaled as $\frac{2 \pi}{k}$ ). Similar waveforms are observed many times in experiments, the reader may see them in $[2,7,14,15]$, for example.

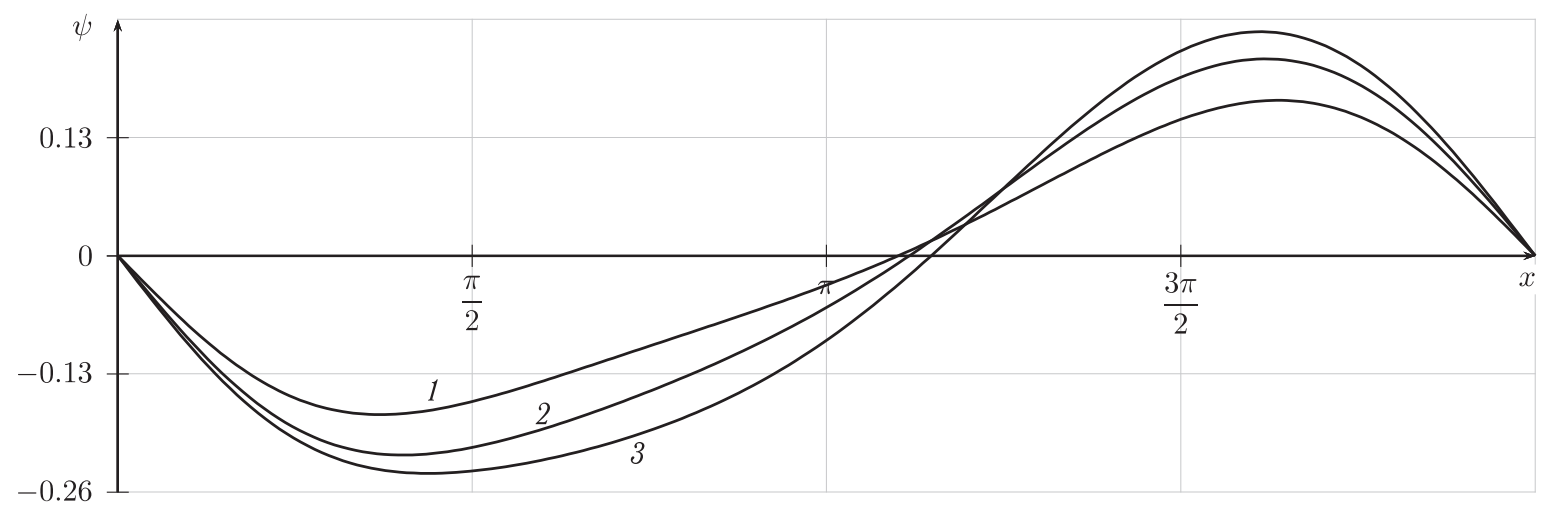

Fig. 3. Waveforms on free surface of water film: $-R e=5 ; 2-R e=7 ; 3-R e=10$

The higher Reynolds' number Re, the higher wave amplitude is (Fig. 4, 5). Wave amplitudes depicted on Fig. 4 were calculated by formula [1, 2]

$$
a=\frac{\delta_{\max }-\delta_{\min }}{\delta_{\max }+\delta_{\min }}
$$

We used the formula $[14,16]$

$$
A=\frac{\delta_{\max }-\langle\delta\rangle}{\langle\delta\rangle}
$$


to calculate wave amplitudes depicted on Fig. 5. By $\langle\delta\rangle$ in (23) the mean liquid film thickness is denoted.

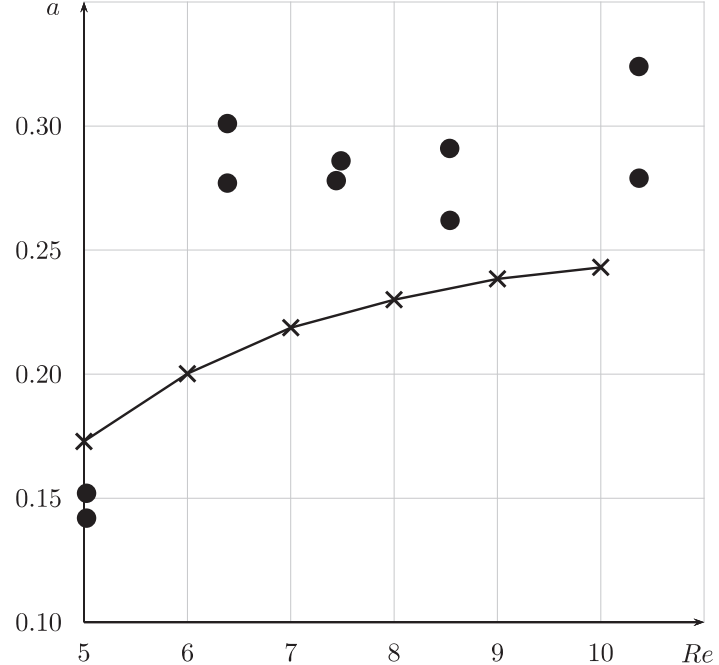

Fig. 4. Amplitudes (water) calculated by $(22): \bullet-[2] ; \times-$ our results

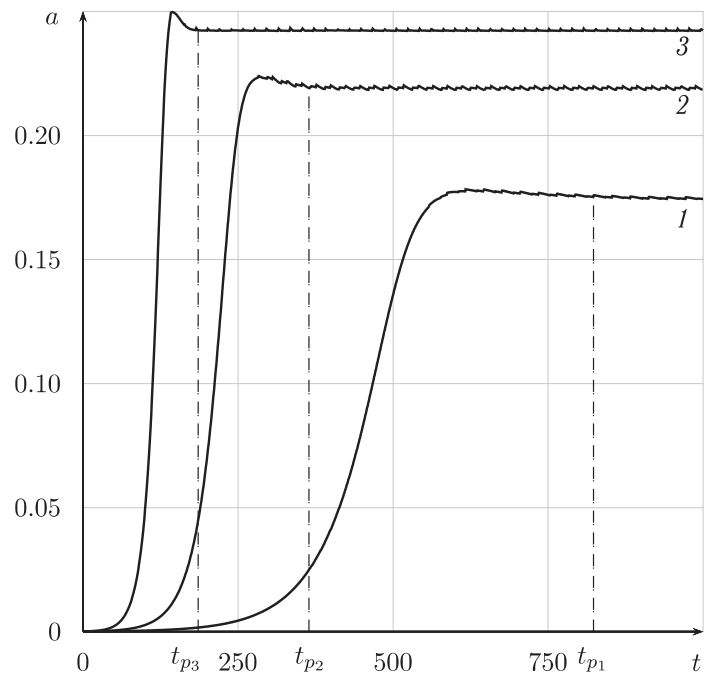

Fig. 6. Amplitude time dependence for water film: $1-R e=5 ; 2-R e=7 ; 3$ $-R e=10$

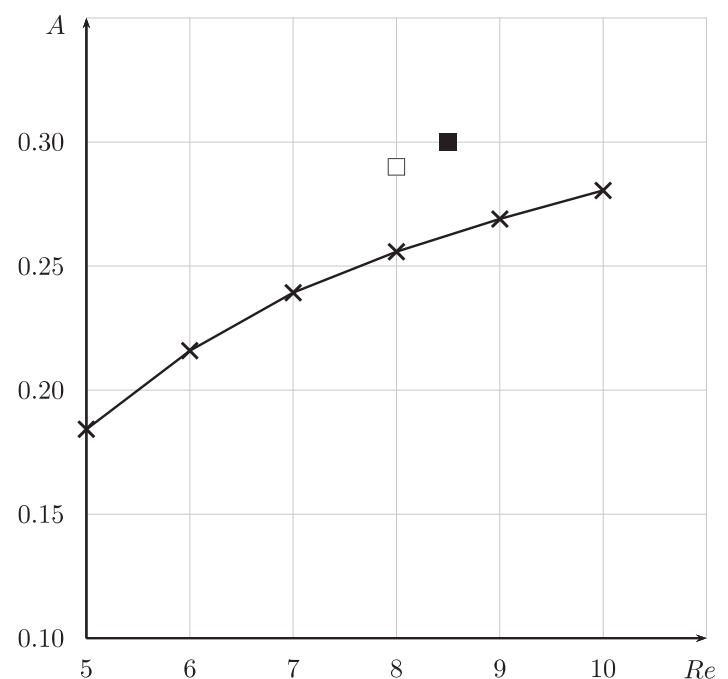

Fig. 5. Amplitudes (water) calculated by (23): $\mathbf{\square}-[14] ; \square-[16] ; \times-$ our results

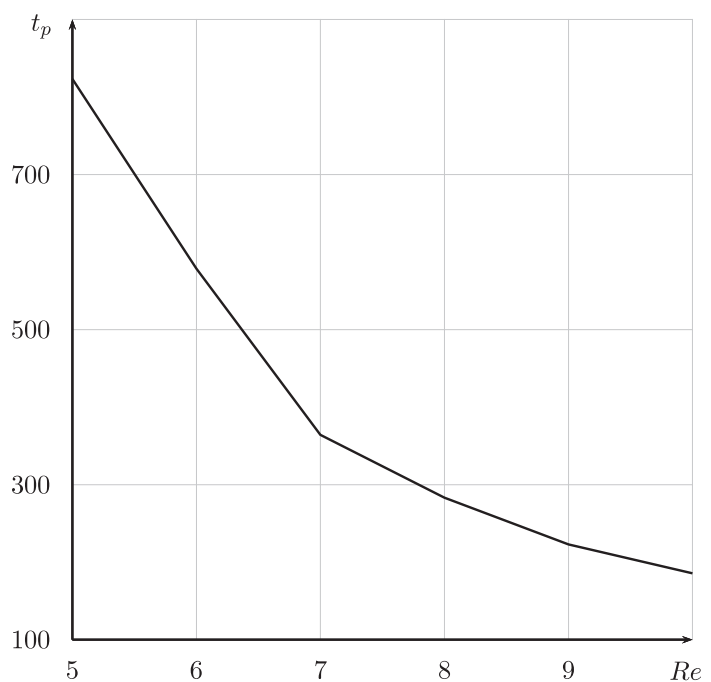

Fig. 7. Duration of regular wave forming for water film

Fig. 6 shows how amplitudes change while regular wavy flows are forming. On Fig. 6 we marked the times $t_{p_{1}}=823,2, t_{p_{2}}=364,3, t_{p_{3}}=185,7$ when the regular wave flows had already formed. As shown on Fig. 7, the higher Reynolds' number Re, the less time $t_{p}$ is required.

\section{References}

1. Kapitza P.L. [Wave Flow of Thin Layer of Viscous Fluid]. Zhurnal Eksperimental'noy $i$ Teoreticheskoy Fiziki, 1948, vol. 18, no. 1, pp. 3-18. (in Russian) 
2. Kapitza P.L., Kapitza S.P. [Wave Flow of Thin Layer of Viscous Fluid]. Zhurnal Eksperimental'noy i Teoreticheskoy Fiziki, 1949, vol. 19, no. 2, pp. 105-120. (in Russian)

3. Vellingiri R. Dynamics of a Liquid Film Sheared by Co-Flowing Turbulent Gas. Int. J. Multiphase Flow, 2013, vol. 56, pp. 93-104. DOI: 10.1016/j.ijmultiphaseflow.2013.05.011

4. Trifonof Y.Y. Stability of the Wavy Film Falling Down a Vertical Plate: The DNS Computations and Floquet Theory. Int. J. Multiphase Flow, 2014, vol. 61, pp. 73-82. DOI: 10.1016/j.ijmultiphaseflow.2014.01.006

5. Dietse G.F., Al-Sibai F., Kneer R. Experimental Study of Flow Separation in Laminar Falling Liquid Films. J. Fluid Mech., 2009, vol. 637, pp. 73-104. DOI: 10.1017/S0022112009008155

6. Dietse G.F., Ruyer-Quil Ch. Wavy Liquid Films in Interaction with a Confined Laminar Gas Flow. J. Fluid Mech., 2013, vol. 722, pp. 348-393. DOI: 10.1017/jfm.2013.98

7. Meza C.E., Balakotaiah V. Modeling and Experimental Studies of Large Amplitude Waves on Vertically Falling Films. Chem. Eng. Sci.,, 2008, vol. 68, no. 19. pp. 4707-4734. DOI: $10.1016 /$ j.ces.2007.12.030

8. Ruyer-Quil Ch., Mannaville P. Improved Modeling of Flows Down Inclined Planes. The European Physical Journal B, 2000, vol. 15, pp. 357-369. DOI: $10.1007 / \mathrm{s} 100510051137$

9. Prokudina L.A., Vyatkin G.P. Self-organization of Perturbations in Fluid Films. Doklady Physics, 2011, vol. 56, no. 8. pp. 444-447. DOI: 10.1134/S1028335811080039

10. Prokudina L.A. Influence of Surface Tension Inhomogeneity on the Wave Flow of a Liquid Film. J. Eng. Phys. Thermophys., 2014, vol. 87, no. 1, pp. 165-173. DOI: $10.1007 / \mathrm{s} 10891-014-0996-2$

11. Prokudina L.A., Vyatkin G.P. Instability of a Nonisothermal Liquid Film. Doklady Physics, 1998, vol. 43, no. 10, pp. 652-654. (in Russian)

12. Prokudina L.A., Salamatov E.A. Shear Stress Influence on Wave Characteristics of Liquid Film. Bulletin of the South Ural State University. Series: Mathematics. Mechanics. Physics, 2012, vol. 7, no. 34, pp. 173-176. (in Russian)

13. Berezin I.S., Zhidkov N.P. Metody vychisleniy [Computing Methods]. Moscow, FIZMATLIT, 1959. 620 p. (in Russian)

14. Rogovaya I.A., Olevskiy V.M., Runova N.G. [Measurement of Parameters of Wavy Liquid Film Flow on Vertical Plate]. Teoreticheskie osnovy khimicheskoi tekhnologii [Theoretical Foundations of Chemical Engineering], 1969, vol. 3, no. 2, pp. 200-208. (in Russian)

15. Lel V.V., Al-Sibai F., Leefken A., Renz U. Local Thickness and Wave Velocity Measurement of Wavy Films with a Chromatic Confocal Imaging Method and a Fluorescence Intensity Technique. Exprements in Fluids, 2005, vol. 39, no. 5, pp. 856-864. DOI: $10.1007 / \mathrm{s} 00348-005-0020-\mathrm{x}$

16. Penev V., Krylov V.S., Boyadjiev Ch., Vorotilin V.P. Wavy Flow of Thin Liquid Films. Int. J. Heat Mass Transfer, 1972, vol. 15, no. 7, pp. 1395-1406. DOI: 10.1016/0017-9310(72)90019-1

Received January 19, 2015 


\title{
МОДЕЛИРОВАНИЕ ВОЛНОВОЙ ПОВЕРХНОСТИ ВЕРТИКАЛЬНОЙ ЖИДКОЙ ПЛЕНКИ ПРИ УМЕРЕННЫХ ЧИСЛАХ РЕЙНОЛЬДСА
}

\author{
Л.А. Прокудина, Е.А. Саламатов
}

\begin{abstract}
В рамках модельного нелинейного дифференциального уравнения, которое описывает эволюцию свободной поверхности вертикальной жидкой пленки, исследовано развитие периодических возмущений на вертикальной пленке воды в диапазоне чисел Рейнольдса $R e \in[5 ; 10]$. Модельное уравнение решалось методом конечных разностей на прямоугольной равномерной сетке. Исследованием роста единичной погрешности получены условия, которым должны удовлетворять параметры расчетной сетки, чтобы погрешности не увеличивались в ходе вычислений. В результате рассчитаны профили волн на свободной поверхности вертикальной пленки воды, время формирования режима стекания с регулярным профилем волны, характер изменения амплитуды периодического возмущения при формировании режима с регулярным профилем волны. Осуществлено сравнение рассчитанных амплитуд с результатами экспериментов.

Ключевые слова: жидкая пленка; амплитуда; профиль волны; нелинейное развитие возмущений.
\end{abstract}

\section{Литература}

1. Капица, П.Л. Волновое течение тонких слоев вязкой жидкости / П.Л. Капица // Журнал экспериментальной и теоретической физики. - 1948. - Т. 18, № 1. - С. 3-18.

2. Капица, П.Л. Волновое течение тонких слоев вязкой жидкости / П.Л. Капица, С.П. Капица // Журнал экспериментальной и теоретической физики. - 1949. - Т. 19, № 2. C. $105-120$.

3. Vellingiri, R. Dynamics of a Liquid Film Sheared by Co-Flowing Turbulent Gas / R. Vellingiri // Int. J. Multiphase Flow. - 2013. - V. 56. - P. 93-104.

4. Trifonof, Y.Y. Stability of the Wavy Film Falling Down a Vertical Plate: The DNS Computations and Floquet Theory / Y.Y. Trifonof // Int. J. Multiphase Flow. - 2014. V. 61. - P. 73-82.

5. Dietse, G.F. Experimental Study of Flow Separation in Laminar Falling Liquid Films / G.F. Dietse, F. Al-Sibai, R. Kneer // J. Fluid Mech. - 2009. - V. 637. - P. 73-104.

6. Dietse, G.F. Wavy Liquid Films in Interaction with a Confined Laminar Gas Flow G.F. Dietse, Ch. Ruyer-Quil // J. Fluid Mech. - 2013. - V. 722. - P. 348-393.

7. Meza, C.E. Modeling and Experimental Studies of Large Amplitude Waves on Vertically Falling Films / C.E. Meza, V. Balakotaiah // Chem. Eng. Sci. - 2008. - V. 68, № 19. P. $4707-4734$.

8. Ruyer-Quil, Ch. Improved Modeling of Flows Down Inclined Planes / Ch. Ruyer-Quil, P. Manneville // The European Physical Journal B. - 2000. - Vol. 15. - P. 357-369.

9. Прокудина, Л.А. Самоорганизация возмущений в жидких пленках / Л.А. Прокудина, Г.П. Вяткин // Доклады АН. - 2011. - Т. 439, № 4. - С. 481-484.

10. Прокудина, Л.А. Влияние неоднородности поверхностного натяжения на волновое течение жидкой пленки / Л.А. Прокудина // Инженерно-физический журнал. - 2014. Т. 87 , № 1. - C. $158-166$. 
11. Прокудина, Л.А. Неустойчивость неизотермической жидкой пленки / Л.А. Прокудина, Г.П. Вяткин // Доклады АН. - 1998. - Т. 362, № 6. - С. 770-772.

12. Прокудина, Л.А. Влияние касательного напряжения на волновые характеристики жидкой пленки / Л.А. Прокудина, Е.А. Саламатов // Вестник ЮУрГУ. Серия: Математика. Механика. Физика. - 2012. - Т. 7, № 34. - С. 173-176.

13. Березин, И.С. Методы вычислений / И.С. Березин, Н.П. Жидков. - М.: ФИЗМАТЛИТ, 1959. - 620 c.

14. Роговая, И.А. Измерение параметров пленочного волнового течения на вертикальной пластине / И.А. Роговая, В.М. Олевский, Н.Г. Рунова // Теоретические основы химической технологии. - 1969. - Т. 3, № 2. - С. 200-208.

15. Local Thickness and Wave Velocity Measurement of Wavy Films with a Chromatic Confocal Imaging Method and a Fluorescence Intensity Technique / V.V. Lel, F. Al-Sibai, A. Leefken, U. Renz // Exprements in Fluids. - 2005. - V. 39, № 5. - P. 856-864.

16. Wavy Flow of Thin Liquid Films / V. Penev, V.S. Krylov, Ch. Boyadjiev, V.P. Vorotilin // Int. J. Heat Mass Transfer. - 1972. - V. 15, № 7. - P. 1395-1406.

Людмила Александровна Прокудина, доктор физико-математических наук, доцент, кафедра «Прикладная математика», Южно-У ральский государственный университет (г. Челябинск, Российская Федерация), prokudina-la@mail.ru.

Евгений Александрович Саламатов, кандидат физико-математических наук, Emerson Process Management, ЗАО «ПГ «Метран» (г. Челябинск, Российская Федерация), salamatov@gmx.com, yevgeny.salamatov@emerson.com.

Поступила в редакиию 19 января 2015 г. 